\title{
Knockdown of DDX46 inhibits proliferation and induces apoptosis in esophageal squamous cell carcinoma cells
}

\author{
BIN LI ${ }^{1,3}$, YU-MIN LI ${ }^{2,3}$, WEN-TING HE ${ }^{2,3}$, HAO CHEN ${ }^{2,3}$, HONG-WEN ZHU ${ }^{3}$, \\ TAO LIU ${ }^{2,3}$, JIAN-HUA ZHANG ${ }^{1}$, TIE-NIU SONG ${ }^{1}$ and YA-LI ZHOU ${ }^{3}$
}

\begin{abstract}
Departments of ${ }^{1}$ Thoracic Surgery and ${ }^{2}$ General Surgery, and ${ }^{3}$ Gansu Provincial Key Laboratory of Digestive System Tumors, Lanzhou University Second Hospital, Lanzhou University Second Clinical Medical College, Lanzhou 730030, P.R. China
\end{abstract}

Received January 28, 2016; Accepted February 24, 2016

DOI: $10.3892 /$ or.2016.4803

\begin{abstract}
Esophageal squamous cell carcinoma (ESCC) is the most common type of esophageal carcinoma and remains the leading cause of cancer-related death worldwide. DEAD-box RNA helicases play critical roles in cellular metabolism and in many cases have been implicated in cellular proliferation and neoplastic transformation. DDX46 belongs to DEAD-box helicase family, the expression pattern of DDX46 in ESCC tissues and the biologic role in ESCC progression have not been implicated previously. In this study, DDX46 expression in human ESCC and adjacent normal tissues were explored using immunohistochemistry, and ESCC cell lines compared with normal esophageal epithelium cell were quantified using real-time PCR. Next, lentivirus-mediated RNA interference was applied to silence DDX46 in TE-1 and Eca-109 cells. Cell growth was monitored using high content screening. Cell viability was measured by MTT assay. Cell colony-forming capacity was measured by colony formation assay. Cell cycle progression and apoptosis were determined by flow cytometry. Further, the stress and apoptosis signaling antibody array kit was used to detect the changes of signaling molecules in TE-1 cells after DDX46 knockdown. We found that DDX46 was significantly upregulated in ESCC tissues and cells compared with normal tissues and cells. DDX46 knockdown led to decreased proliferation and increased apoptosis in TE-1 and Eca-109 cells. Moreover, DDX46 silencing resulted in apoptotic induction via decreased phosphorylation of Akt and I $\mathrm{B} \alpha$, as well as negative regulation of NF- $\mathrm{B}$ signaling. In conclusion, these results demonstrate that DDX46 knockdown inhibited cell growth, and induced apoptosis, suggest that
\end{abstract}

Correspondence to: Professor Yu-Min Li, Department of General Surgery, Gansu Provincial Key Laboratory of Digestive System Tumors, Lanzhou University Second Hospital, Lanzhou University Second Clinical Medical College, 82 Cuiyingmen, Chengguan, Lanzhou, Gansu 730030, P.R. China

E-mail: liym@lzu.edu.cn

Key words: DDX46, RNAi, esophageal squamous cell carcinoma, proliferation, apoptosis
DDX46 is critical for ESCC cells proliferation. In addition, this study provides a foundation for further study into the clinical potential diagnosis and novel therapeutic target for ESCC.

\section{Introduction}

Esophageal carcinoma is the eighth most common malignancy worldwide, with an estimated 455,800 new cases and 400,200 deaths annually (1), $70 \%$ of which occurred in China (2). Approximately $60 \%$ of all patients have advanced disease at the time of diagnosis, although advances in surgical techniques and neoadjuvant and adjuvant chemoradiotherapy have led to increasingly multimodal therapy for patients, the longevity remains disappointingly low, with 5 -year overall survival uniformly $\sim 15 \%(1,2)$. The two main histological types: esophageal squamous cell carcinoma (ESCC) and adenocarcinoma (EAC), exhibit many differences in etiology, epidemiology, genetic landscape, clinical characteristics and prognosis $(3,4)$, while $>90 \%$ of cases in China is ESCC (2). The application of targeted therapy is mostly limited to EAC, few patients with advanced ESCC can be cured with synthetic treatment modality (5). Therefore, it is important to discover the molecular mechanism driving ESCC progression and develop novel diagnostic and prognostic methods and potential therapeutic targets.

RNA plays a central role in transmitting of genetic information from DNA to protein. RNA helicases are a large family of enzymes that perform diverse cellular functions in virtually all steps of RNA metabolism, ranging from gene transcription to RNA decay (6). DEAD-box helicases form the largest family in RNA helicases and are ubiquitous from bacteria to humans, play key roles in rearranging RNA-RNA and RNA-protein interactions, participate in almost all aspects of processes, including pre-mRNA splicing, ribosome biogenesis, RNA transport, mRNA export, translation initiation and termination, and organelle gene expression (7). In addition, they also participate in an ATPase-independent manner in basic processes such as transcriptional regulation (7). Several DEAD-box RNA helicases are aberrantly expressed in tumor tissues and cell lines, and involved in the processes that are key to cellular proliferation and cancer progression and transformation, therefore, it is not surprising that deregulation of expression or function of these proteins has been implicated 
in cancer development or progression (8). These proteins are excellent potential tumor-specific vulnerabilities that one might be able to capitalize upon for the development of novel molecular-targeted therapies $(9,10)$.

DDX46 gene, also named as PRPF5 or Prp5, belongs to DEAD-box RNA helicase family. In vitro biochemical assays have shown that DDX46 is required for stable association of $\mathrm{U} 2$ in the pre-spliceosome and have multiple functions in nuclear pre-mRNA splicing before or during pre-spliceosome assembly $(11,12)$. In vivo zebrafish studies have shown that DDX46 is required for the development of the digestive organs and brain through the control of pre-mRNA splicing (13), and multi-lineage differentiation of hematopoietic stem cells through the regulation of specific gene expressions (14). Human DDX46 gene is located in chromosome 5q31.1 (15). DDX46 has not been reported involved in human cancers, except that $\mathrm{Li}$ and colleagues (16) reported that DDX46 expression was upregulated in colorectal carcinoma tissues and critical for colorectal carcinoma cell proliferation. The clinical significance and biologic role of DDX46 in ESCC, however, have not been implicated previously. In this study, we found that DDX46 was overexpressed in human ESCC tissues as compared with adjacent normal tissues, and silencing of DDX46 expression inhibited proliferation and induced apoptosis of human ESCC cells in which DDX46 was amplified.

\section{Materials and methods}

Patients and tissue specimens. All tissue specimens were obtained from the 69 ESCC patients who underwent esophagogastrostomy from January 2014 to December 2014 at the Department of Thoracic Surgery, Lanzhou University Second Hospital (Lanzhou, China). For histological evaluation, hematoxylin-eosin stained sections from each specimen were determined by pathologists. Preoperative clinical evaluation was performed for all patients consisting of ultrasonography of the neck, CT scan of the chest and abdomen, barium swallow examination, upper gastrointestinal endoscopy with biopsy, $\mathrm{Tc}^{99 \mathrm{~m}}$ whole body bone scan. No patient received chemo- or radiotherapy prior to operation. All of the specimens had matched adjacent normal tissues. The study protocol was approved by the medical ethics committee of our hospital.

Immunohistochemistry. Immunohistochemistry (IHC) was performed using the immunohistochemical SP kit (Maixin Biotech, Fuzhou, China) according to the manufacturer's instructions. Briefly, paraffin-embedded tissue sections were deparaffinized and rehydrated. Endogenous peroxidase activity was blocked by incubation in $3 \%$ hydrogen peroxide for $15 \mathrm{~min}$ at room temperature. Antigen was retrieved by heating in a pressure cooker for $5 \mathrm{~min}$ in sodium citrate buffer (pH 6.0). Non-specific antigens were blocked by incubating in sheep serum for $30 \mathrm{~min}$ at room temperature. After incubation with diluted rabbit anti-DDX46 (1:1,000 dilution, SigmaAldrich, St. Louis, MO, USA) overnight at $4^{\circ} \mathrm{C}$, the sections were rinsed with PBS and secondary antibodies were applied at a 1:500 dilution in PBS for $30 \mathrm{~min}$ at room temperature. Then the sections were incubated with streptavidin-horseradish peroxidase (HRP) for $30 \mathrm{~min}$ at room temperature and rinsed with PBS and incubated for 15 min with the chromogen diami- nobenzidine (DAB) and counterstained with hematoxylin. The images of sections were captured under a transmission light microscope.

DDX46 expression was quantified using a visual grading system based on the extent of staining (graded from 0 to 4 : 0 , none; $1,1-25 \% ; 2,26-50 \% ; 3,51-75 \% ; 4,>75 \%)$ and the intensity of staining (graded from 0 to 3 : 0 , no staining; 1, weak staining; 2, moderate staining; 3 , strong staining). The index value was calculated as a product of the extent of staining and intensity of staining scores to evaluate the expression of DDX46. DDX46 expression was classified into three grades: negative (index values 0-3), weak positive (index values 4-8) and strong positive (index values 9-12).

Cell culture and lentivirus transfection. The human ESCC cell lines (TE-1, Eca-109, TE-11 and KYSE-150) were purchased from the Cell Bank of the Chinese Academy of Sciences (Shanghai, China), the human normal esophageal epithelium cell line Het-1A was purchased from the Bioleaf Biotech (Shanghai, China). All cell lines tested negative for any mycoplasma contamination, and were maintained in a standard humidified incubator at $37^{\circ} \mathrm{C}$ in $5 \% \mathrm{CO}_{2}$ and used at passage numbers 5-7 for the experiments.

Using specific short hairpin RNA (shRNA) mediated by lentiviral vector (LV) to knockdown DDX46. Lentiviral constructs expressing DDX46 shRNA (DDX46-shRNA-LV) and non-silencing negative control LV (Control-LV) were designed and synthesized by Genechem Chemtech (Shanghai, China) according to the sequence encoding human DDX46 complementary DNA (NM_014829). The silencing shRNA sequence was 5'-CATCCAAACCCAAGCTATT-3', and nonsilencing negative control sequence was 5'-TTCTCCGA ACGTGTCACGT-3'.

For lentivirus transfection, TE-1 and Eca-109 cells were cultured in 6-well plates at a concentration of $2 \times 10^{5}$ cells per well on the day before shRNA transfection. Then, DDX46shRNA-LV was transfected into cells at a multiplicity of infection (MOI) of 5 (TE-1) or 10 (Eca-109) using polybrene $(10 \mu \mathrm{g} / \mathrm{ml})$ and enhanced infection solution (Genechem Chemtech). At the same time, Control-LV was transfected into cells using the same methods to control for the impact of the viral vector. After incubation for $12 \mathrm{~h}$, the medium was replaced with fresh L-15 medium. After $72 \mathrm{~h}$ of incubation, cells were observed under fluorescence microscopy (MicroPublisher 3.3RTV; Olympus, Tokyo, Japan) to evaluate the efficiency of transfection. At the indicated time-points, the cells were harvested for mRNA and protein analysis as well as other assays.

Quantitative real-time PCR. The total RNA of cultured cells was isolated using TRIzol reagent (Invitrogen, Carlsbad, CA, USA) according to the manufacturer's instructions. The primers were designed by Beacon Designer 2 software (Premier Biosoft, Palo Alto, CA, USA). DDX46 primers were as follows: forward, 5'-AAAATGGCGAGAAGAGCAACG-3'; and reverse, 5'-CATCATCGTCCTCTAAACTCCAC-3'; glyceraldehyde3-phosphate dehydrogenase (GAPDH) was used as an internal control: forward, 5'-TGACTTCAACAGCGACACCCA-3'; and reverse, 5'-CACCCTGTTGCTGTAGCCAAA-3'). Quantitative real-time PCR (qRT-PCR) was performed with the 7500 Real- 
Time PCR system (Applied Biosystems, Foster City, CA, USA) using the SYBR Green Real-Time PCR assay kit (Takara, Otsu, Japan). The relative expression levels of mRNAs were calculated using the $\Delta \mathrm{Ct}(\Delta \mathrm{Ct}=\mathrm{Ct}$ sample $-\mathrm{Ct}$ control $)$ or $2^{-\Delta \Delta \mathrm{Ct}}(\Delta \Delta \mathrm{Ct}=\Delta \mathrm{Ct}$ sample $-\Delta \mathrm{Ct}$ control $)$ method.

Western blot analysis. The total protein was extracted from cultured cells using total protein extraction kit as recommended by the manufacturer (Sigma-Aldrich). The protein extracts $(20 \mu \mathrm{g})$ were separated on $10 \%$ sodiumdodecyl sulfate-polyacrylamide gels (SDS-PAGE; Tanon Technology, Shanghai, China) and electrotransfered onto polyvinylidene difluoride (PVDF) membranes (Millipore, Billerica, MA, USA). The membranes were blocked in Tris-buffered saline with Tween-20 (TBST) containing 5\% powdered milk for $1 \mathrm{~h}$ at room temperature, then incubated overnight at $4^{\circ} \mathrm{C}$ in blocking solution with primary antibody as follows: anti-DDX46 (1:1,000 dilution, Sigma-Aldrich); anti-GAPDH (used as a loading control, 1:2,000 dilution, Santa Cruz Biotechnology, Santa Cruz, CA, USA). After washing with TBST, the membranes were incubated with HRP-conjugated secondary antibody (1:2,000 dilution, Beyotime, Haimen, China) for $2 \mathrm{~h}$ at room temperature. The membranes were then visualized using Pierce ECL Western Blotting Substrate (Thermo Fisher Scientific, Rockford, IL, USA) according to the manufacturer's instructions.

Cell growth assay. The cultured cells were seeded in 96-well plates at a density of $2 \times 10^{3}$ cells per well. Dynamic growth of green fluorescence protein (GFP)-labeled cells were monitored once a day for 5 consecutive days using High Content Screening (HCS) (Cellomics, ArrayScan VT1; Thermo Fisher Scientific). Cell growth curves were drawn.

MTT cell viability assay. After the indicated treatments, the cells in the logarithmic growth phase were seeded in 96-well plates in triplicate at densities of $2 \times 10^{3}$ cells per well. Cell proliferation was evaluated at 1,2,3,4 and 5 days using the methylthiazoltetrazolium (MTT) assay. In brief, at indicated time-points, cells were incubated with $20 \mu \mathrm{l}$ sterile MTT dye ( $5 \mathrm{mg} / \mathrm{ml}$, Sigma-Aldrich) for $4 \mathrm{~h}$ at $37^{\circ} \mathrm{C}$, followed by removal of the culture medium and addition of $100 \mu \mathrm{l}$ dimethyl sulfoxide (DMSO) to each well to stop the reaction. Then the 96-well plates were shaken for $5 \mathrm{~min}$, and the optical density (OD) values were measured at $490 \mathrm{~nm}$ using a microplate reader (Bio-Rad, Hercules, CA, USA).

Colony formation assay. Cells were plated in 6-well plates at 600 cells per well and cultured for 15 days. After fixation with $4 \%$ paraformaldehyde, the colonies were stained with Giemsa for $15 \mathrm{~min}$. Then the colonies were photographed and counted under a microscope. Colonies consisting of $\geq 50$ cells were counted as a clone.

Flow cytometry analysis. For detection of cell apoptotic activity, the cells were cultured in 6-well plates. When the cells reached $\sim 85 \%$ confluence, they were collected and stained using the Annexin V-Allophycocyanin (APC) single-staining Apoptosis Detection kit (eBioscience, San Diego, CA, USA) according to the manufacturer's instructions. For detection of
Table I. Overexpression of DDX46 detected in ESCC tissues by IHC examination.

\begin{tabular}{|c|c|c|c|c|}
\hline & $\begin{array}{c}\text { All } \\
\text { cases }\end{array}$ & Negative & $\begin{array}{l}\text { Weak } \\
\text { positive }\end{array}$ & $\begin{array}{l}\text { Strong } \\
\text { positive }\end{array}$ \\
\hline ESCC tissues & 69 & 7 & 30 & 32 \\
\hline $\begin{array}{l}\text { Adjacent normal } \\
\text { tissues }\end{array}$ & 69 & 53 & 9 & 7 \\
\hline P-value & \multicolumn{4}{|c|}{$<0.001$} \\
\hline
\end{tabular}

the cell cycle, the cells were cultured in 6 -cm dishes. At $~ 85 \%$ confluence, cell cycle analysis was performed after staining with propidium iodide (Sigma-Aldrich). Both apoptosis and cell cycle distribution were quantified using a Guava easyCyte HT flow cytometer (Millipore).

Stress and apoptosis signaling antibody array. At $~ 85 \%$ confluence, cell extracts were prepared and analyzed using the PathScan ${ }^{\circledast}$ Stress and Apoptosis Signaling Antibody Array kit analysis (chemiluminescent readout, Cell Signaling Technology, Danvers, MA, USA) according to the manufacturer's instructions. Images were acquired by briefly exposing the slide to standard chemiluminescent film.

Statistical analysis. All experiments were performed in triplicate. The data are expressed as means \pm standard deviation (SD) determined from three independent experiments. Student's t-test or Mann-Whitney U test was applied for statistical analysis using SPSS software (version 16.0; SPSS Inc., Chicago, IL, USA). P-value $<0.05$ was considered statistically significant.

\section{Results}

DDX46 is overexpressed in ESCC tissues and cell lines. DDX46 protein expression was examined in 69 ESCC tissues and adjacent normal tissues using IHC. It showed that DDX46 expression was predominantly located in the nucleus of ESCC cells, and comparative analysis revealed that the expression levels of DDX46 in ESCC tissues were higher than those in matched adjacent non-tumor tissues $(\mathrm{P}<0.001$; Table I and Fig. 1). qRT-PCR analysis showed that DDX46 mRNA were significantly upregulated in all four tested human ESCC cell lines (TE-1, Eca-109, TE-11 and KYSE-150), compared with the human normal esophageal epithelium cell line Het-1A (Fig. 2). These results suggest that DDX46 expression was upregulated in ESCC.

Lentivirus-mediated RNAi efficiently decreases DDX46 expression. TE-1 and Eca-109 cells were chosen to silence the endogenous DDX46 expression by DDX46-shRNA-LV. The transfection efficiency of LV was confirmed by observing GFP-expressing cells under a fluorescence microscope $72 \mathrm{~h}$ after incubation. As shown in Fig. 3A and D, $>90 \%$ of the TE- 1 and Eca-109 cells were transfected. Then the silencing effect of LV in TE-1 and Eca-109 cells was measured by qRT-PCR and western blot analysis. Compared with the Control-LV 


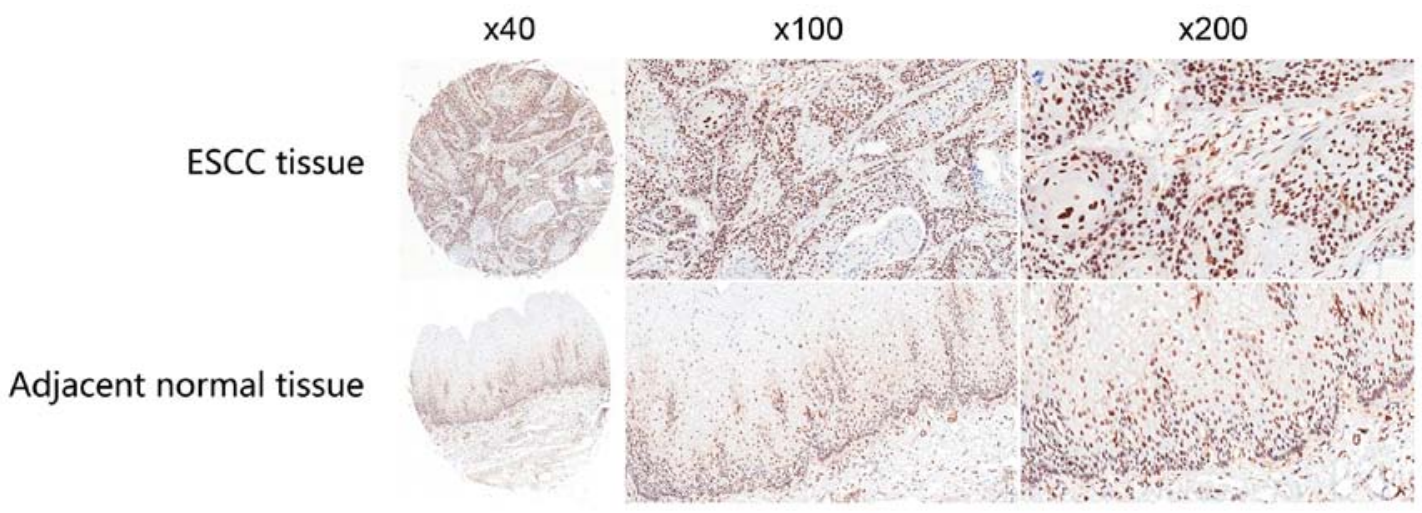

Figure 1. IHC examination shows that DDX46 protein expression was increased in human ESCC tissues, compared with the adjacent normal tissues (magnification, x40, x100 and x200, respectively).

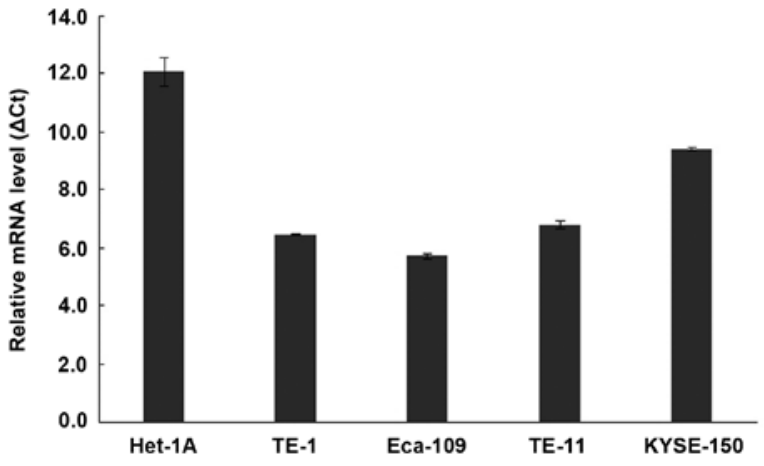

Figure 2. qRT-PCR analysis showing that DDX46 mRNA expression was upregulated in all four tested human ESCC cell lines (TE-1, Eca-109, TE-1 1 and KYSE-150), compared with the normal esophageal epithelium cell line Het-lA. Expression levels were normalized with GAPDH. group, DDX46 mRNA expression level was reduced by $84.6 \%$ (Fig. 3B, P<0.01) and 91.8\% (Fig. 3E, $\mathrm{P}<0.01$ ) in TE-1 and Eca-109 cells, respectively, after DDX46-shRNA-LV transfection. In parallel, DDX46 protein expression level was also significantly decreased in the DDX46-shRNA-LV transfected TE-1 and Eca-109 cells (Fig. 3C and F). These results indicated that the lentivirus-mediated RNAi system was able to knock down the endogenous expression of DDX46 in TE-1 and Eca-109 cells specifically and effectively.

Silencing DDX46 significantly inhibits ESCC cell proliferation. In order to determine the effects of DDX46 on cell growth, cells with green fluorescence were counted once a day for 5 consecutive days using HCS (Fig. 4A and D). The number of GFP-labeled cells in the DDX46-shRNA-LV group
A

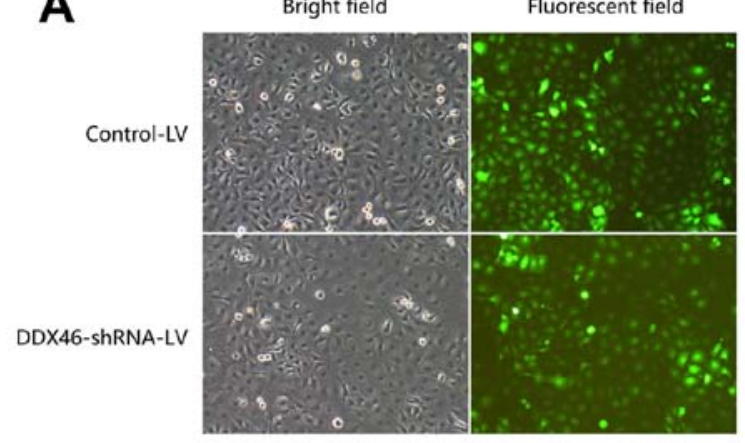

D

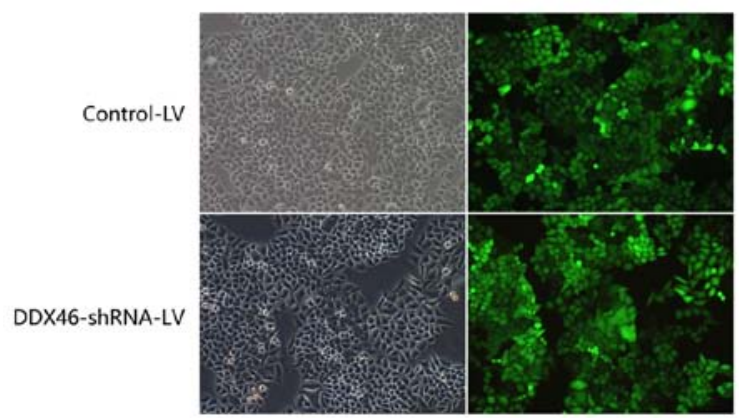

B

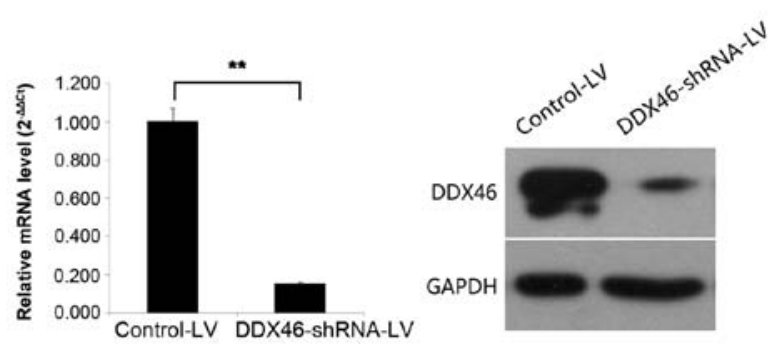

$\mathbf{E}$

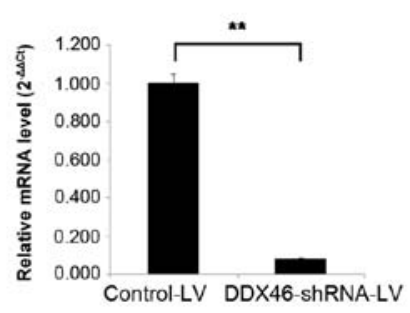

C

$\mathbf{F}$

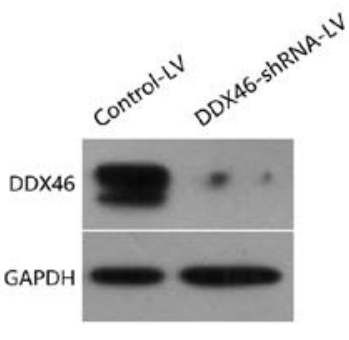

Figure 3. Lentivirus-mediated RNAi efficiently decreases DDX46 expression in ESCC cell lines TE-1 (A-C) and Eca-109 (D-F). (A and D) Transfection efficiency monitored under fluorescence microscopy (magnification, x100). (B and E) qRT-PCR analysis. (C and F) Western blot analysis. Expression levels were normalized with GAPDH. ${ }^{* *} \mathrm{P}<0.01$ versus Control-LV group. 

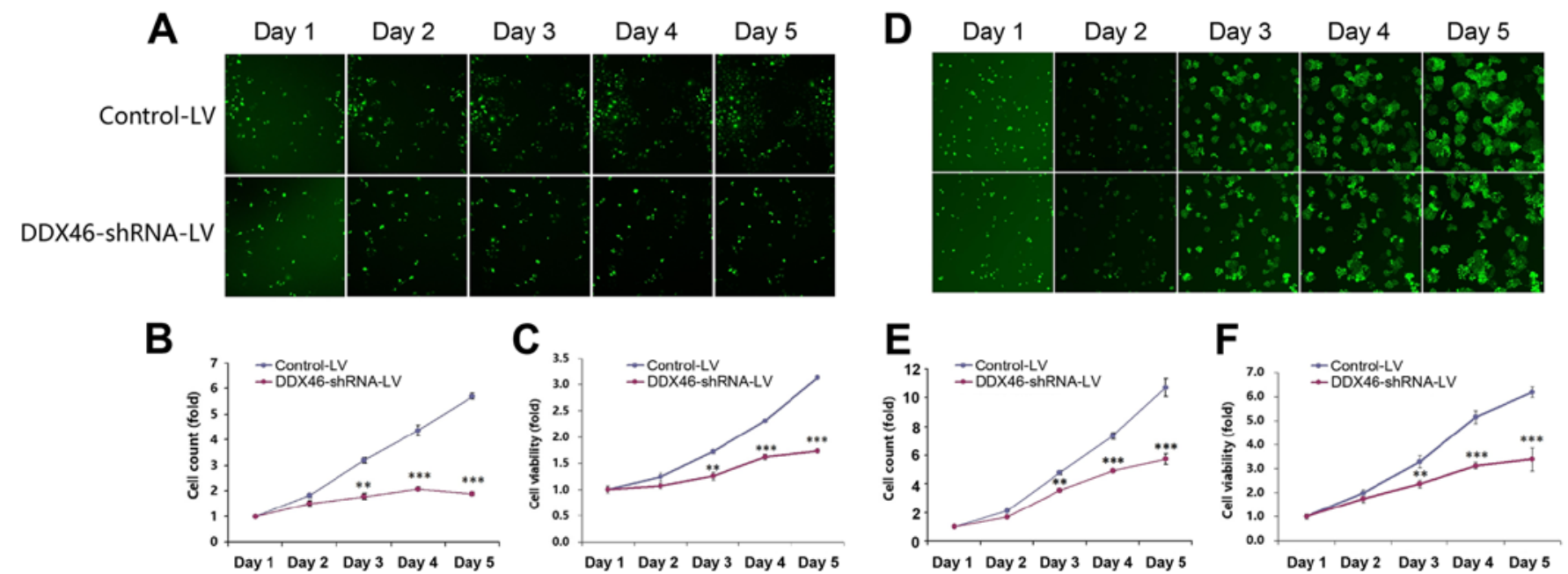

Figure 4. Silencing DDX46 inhibits proliferation of TE-1 (A-C) and Eca-109 (D-F) cells. (A and D) Dynamic growth of GFP-labeled TE-1 and Eca-109 cells transfected with Control-LV and DDX46-shRNA-LV was monitored once a day for 5 consecutive days using HCS (magnification, x50); (B and E) GFP-labeled transfected-TE-1 and Eca-109 cells were counted and cell growth curves were drawn accordingly. (C and F) Effect of DDX46 silencing on the viability of transfected-TE-1 and Eca-109 cells was analyzed by MTT assay, ${ }^{* *} \mathrm{P}<0.01,{ }^{* * *} \mathrm{P}<0.001$ versus Control-LV group.

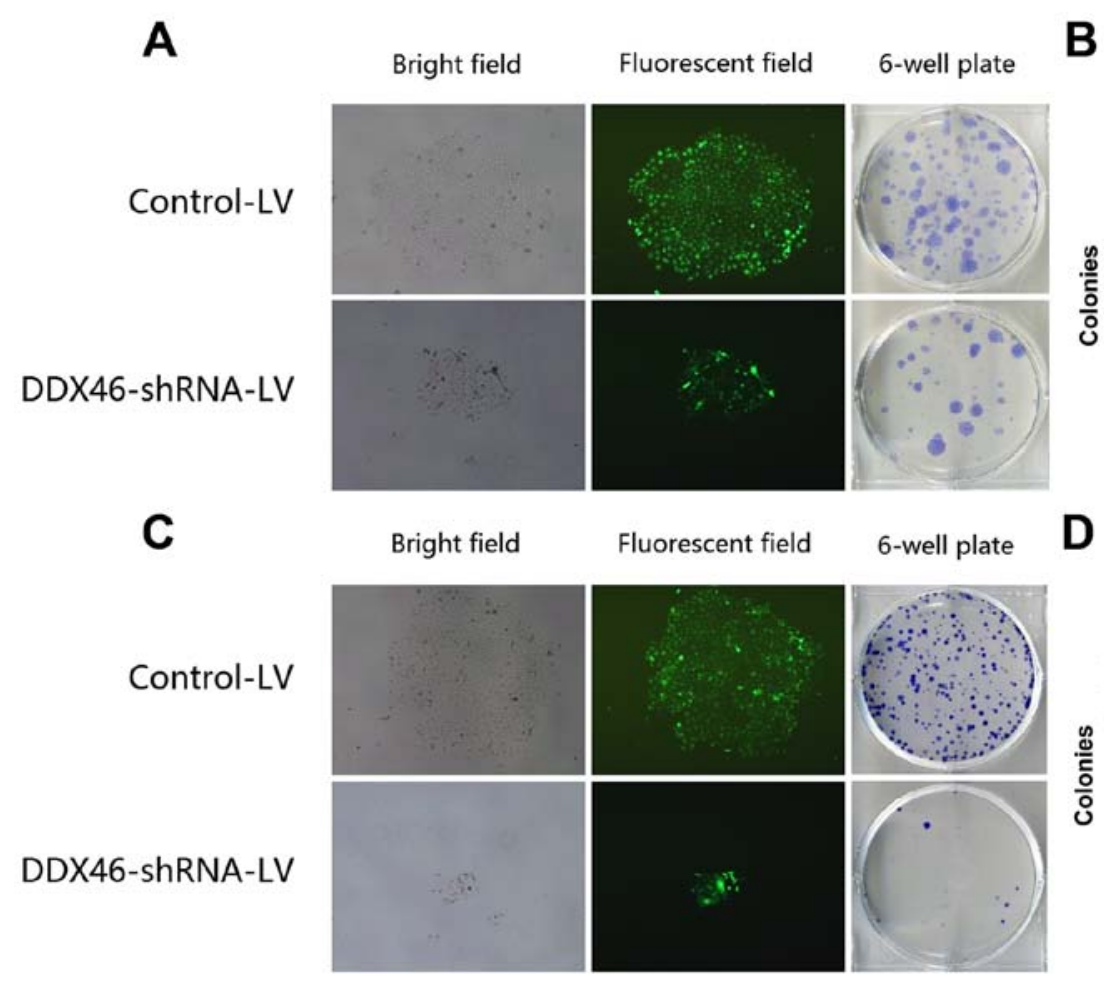

B
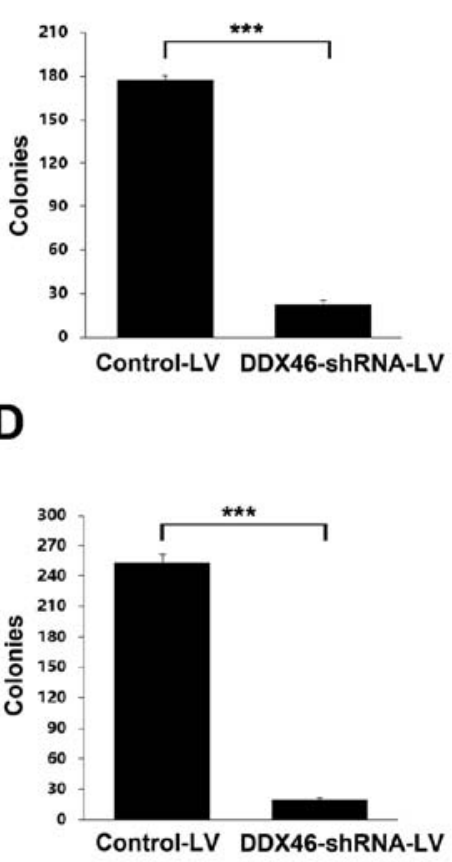

Figure 5. Silencing DDX46 suppresses colony formation capacity of TE-1 (A and B) and Eca-109 (C and D) cells, ${ }^{* * *} \mathrm{P}<0.001$ versus Control-LV group.

was greatly decreased compared to the Control-LV group in both TE-1 (Fig. 4B) and Eca-109 (Fig. 4E) cells. Silencing DDX46 also significantly inhibited cell viability that was analyzed by MTT assays. As illustrated in Fig. 4C and F, cell viability of DDX46-shRNA-LV transfected TE-1 and Eca-109 cells were significantly reduced in a time-dependent manner. Additionally, DDX46 knockdown significantly suppressed colony formation capacity in ESCC cells. The number and size of colonies in both DDX46-shRNA-LV transfected TE-1 and Eca-109 cells were significantly reduced compared with the corresponding Control-LV group (Fig. 5). Collectively, these results suggested that overexpression of DDX46 played an essential role in proliferation of ESCC cells in vitro.

Silencing DDX46 significantly induces apoptosis and regulated cell cycle arrest in ESCC cells. Transfection of DDX46-shRNA-LV resulted in a significant increase in the percentage of apoptotic cells, compared with the Control-LV, in TE-1 (Fig. 6A) and Eca-109 (Fig. 6B) cells. Furthermore, flow cytometry assay indicated that silencing of DDX46 

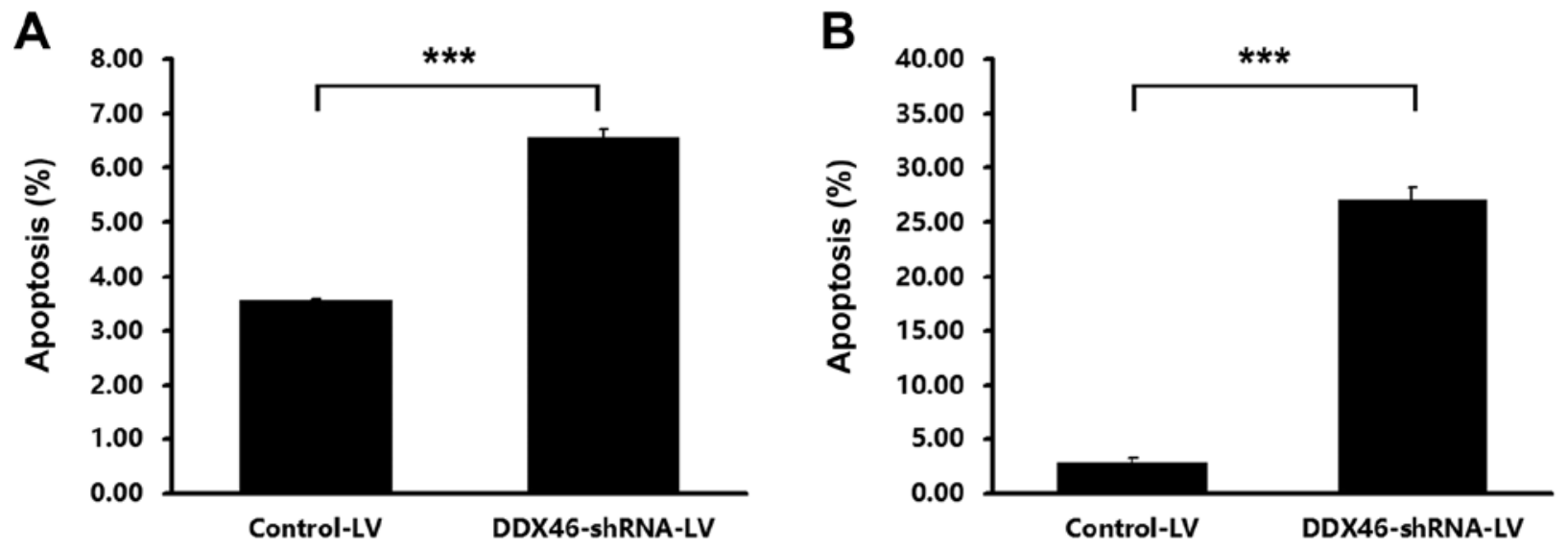

Figure 6. Silencing DDX46 promoted apoptosis of TE-1 (A) and Eca-109 (B) cells, ${ }^{* * * *} \mathrm{P}<0.001$ versus Control-LV group.
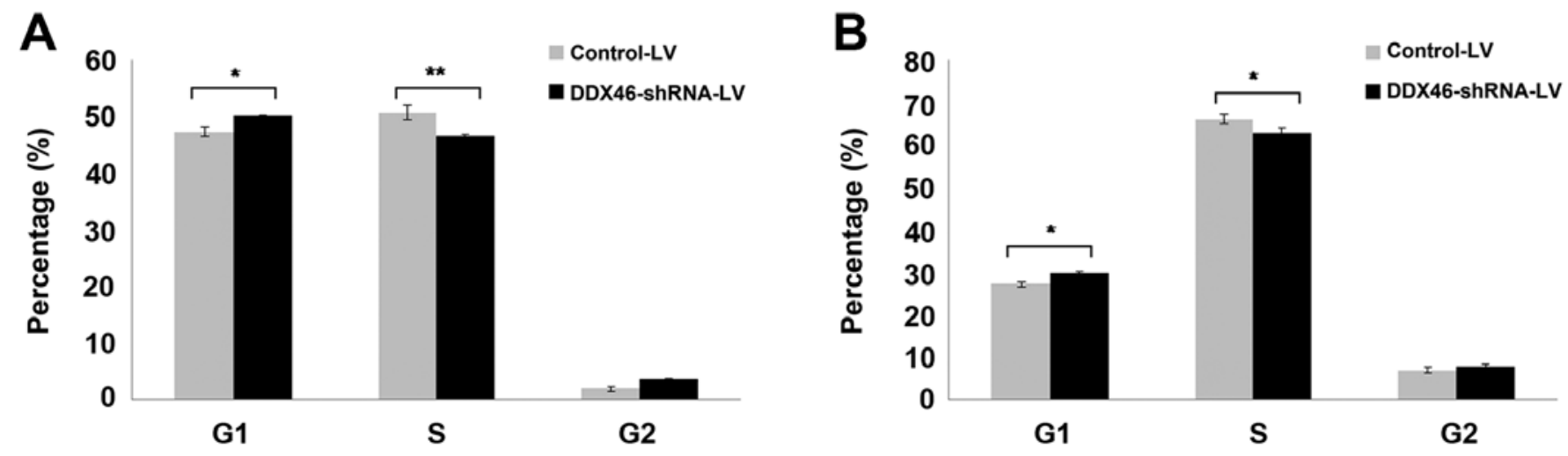

Figure 7. Silencing DDX46 increased the percentage of Gl-phase cells and decreased the percentage of S-phase cells in TE-1 (A) and Eca-109 (B) cells, ${ }^{*} \mathrm{P}<0.05$, ${ }^{* *} \mathrm{P}<0.01$ versus Controi-LV group.

Control-LV

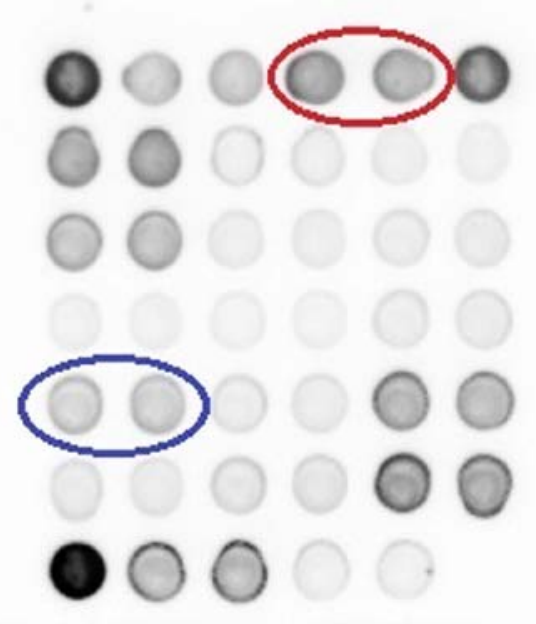

DDX46-ShRNA-LV

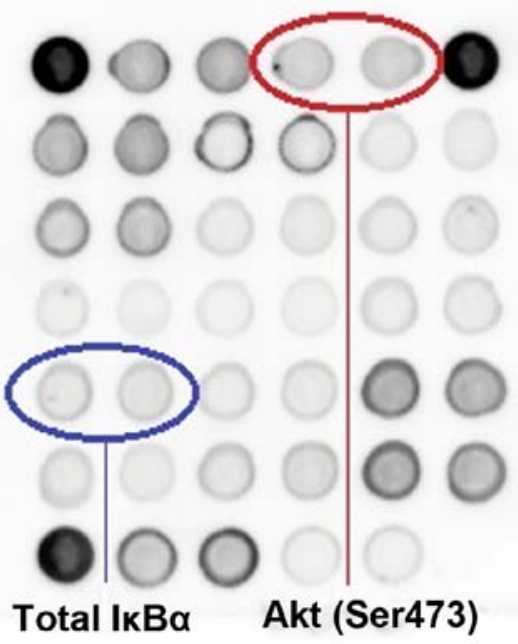

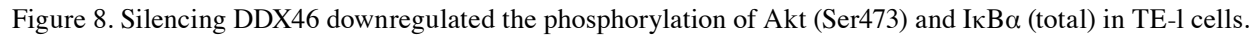

expression markedly increased the percentage of G1-phase cells and decreased the percentage of S-phase cells in TE-1 (Fig. 7A) and Eca-109 (Fig. 7B) cells, the cells were arrested in the G1-phase after knockdown of DDX46. The above data indicated that DDX46 was involved in the regulation of ESCC cell apoptosis and cell cycle progression. 
To further illuminate the molecular mechanisms by which DDX46 regulated ESCC cell apoptosis, the stress and apoptosis signaling antibody array kit was used to detect the changes of signaling molecules in TE-1 cells with or without DDX46 knockdown. As shown in Fig. 8, the phosphorylation of Akt (Ser473) and $\mathrm{I} \kappa \mathrm{B} \alpha$ (total) were downregulated in DDX46-silenced cells, respectively. The data indicated that DDX46 knockdown could significantly induce apoptosis of DDX46 cells via blockade of Akt and I $\mathrm{B} \alpha$ activations.

\section{Discussion}

In this study, we found that DDX46 protein expression in clinical specimens from ESCC tissues was upregulated compared with matched adjacent non-tumor tissues; the expression of DDX46 mRNA was significantly higher in all of the tested human ESCC cell lines compared to the normal esophageal epithelium cell Het-1A. In addition, silencing DDX46 inhibited proliferation and induced apoptosis of ESCC cells. These data indicated that DDX46 overexpression played essential roles in the tumorigenicity of human ESCC.

DEAD-box RNA helicases was first described in 1989 (17), since then it has gradually formed the largest helicases family (7). Members of this family share a conserved core containing nine conserved motifs, including the characteristic D-E-A-D (Asp-Glu-Ala-Asp, or DEAD in one-letter code) motif, which gave the protein family its name; these motifs confer the ATP hydrolysis and RNA unwinding activities that have established them as RNA helicases (8). DEAD-box RNA helicases play key, and often essential, roles in RNA metabolism. Overexpression of these helicases can indicate increased mistakes during RNA and protein production, possibly leading to carcinogenesis. A more recent study of breast cancer gene expression and tissue microarrays, which showed that DDX1 mRNA overexpression and elevated cytoplasmic DDX1 protein were associated with early recurrence and suggested that DDX1 may be an independent prognostic marker for early recurrence in breast cancer (18). DDX6 was first suggested to be involved in tumor development through its cloning as an RNA helicase gene from a human lymphoid cell line with chromosomal breakpoint 11q23.3 (19). Subsequent reports demonstrated that DDX6 protein was elevated in glioblastoma, rhabdosarcoma, lung cancer (20), colorectal carcinoma $(21,22)$ and hepatitis $\mathrm{C}$ virus-related hepatocellular carcinoma (23) as compared with normal tissue. The first report of aberrant DDX5 expression in cancer came from a study which showed that DDX5 was overexpressed and abnormally modified by polyubiquitylation in colorectal carcinoma (24). This was followed by several other reports showing DDX5, and in several cases also DDX17, overexpression in a series of cancers including colon $(25,26)$, prostate $(27)$, breast $(28,29)$, cutaneous squamous cell carcinoma (30) and glioma (31). DDX39 was overexpressed in lung squamous cell carcinomas (32). Recent proteomic study of gastrointestinal stromal tumors correlate to elevated DDX39 levels with an increased risk of metastasis following surgery (33). However, the expression of DDX46 in human cancer tissues remains largely unknown, only Li and colleagues (16) reported on colorectal carcinoma. For the first time, our study assessed DDX46 expression in human ESCC tissues by IHC.
Our results showed that the cellular localization of DDX46 was focused in the nucleus of cells, and DDX46 was more strongly and highly stained in ESCC tissues than in matched normal tissues. In vitro, silencing DDX46 inhibited cell proliferation and colony formation by inducing cell apoptosis and G1-phase cell cycle arrest. These results are similar to a previous study (16), indicating a common role of DDX46 in cancer cell proliferation.

Although numerous reports implied an involvement of DEAD-box RNA helicases in cancer development, the precise mechanism of its contribution to cancer was concealed at the time. Forced overexpression of DDX6 in colorectal carcinoma cells stimulated Tcf transcriptional activity and expression of Wnt pathway target genes (34). DDX5 and DDX17 regulated the $\beta$-catenin pathway by formed complexes with $\beta$-catenin and promoted the ability of $\beta$-catenin to activate gene transcription (26). Some DEAD-box RNA helicases were coactivators of tumor development transcription factors and regulated the expression of downstream molecules. For instance, DDX5 selectively activated p53-dependent p21 expression, thereby influencing entry into cell cycle arrest and apoptosis after DNA damage (35). Whether DDX46 regulates these signaling pathways or others needs further exploration. Herein, we found that DDX46 may play an important role in ESCC cells proliferation by inducing cell apoptosis and cell cycle arrest in vitro. Silencing DDX46 significantly impaired ESCC cell growth, blocked G0-G1 phase progression, and increased the number of apoptotic cells in both TE-1 and Eca-109 cells. The stress and apoptosis signaling antibody array displayed that DDX46 knockdown significantly reduced the phosphorylation of Akt and $\mathrm{I} \kappa \mathrm{B} \alpha$. Akt plays a central role in tumorigenesis, its function is dictated by phosphorylation status (36). Suppressed Akt activity could inhibit proliferation and induce apoptosis (37). Activated Akt could stimulate the phosphorylation and impact various downstream targets, including $\mathrm{I} \kappa \mathrm{B} \alpha$ (38), which is endogenous inhibitor of $N F-\kappa B . N F-\kappa B$ is a nuclear transcription factor that regulates expression of a large number of genes that are critical for the regulation of apoptosis and tumorigenesis. In its inactive form, $N F-\kappa B$ is sequestered in the cytoplasm, bound by members of the $\mathrm{I} \kappa \mathrm{B}$ family of inhibitor proteins, which include $\mathrm{I} \kappa \mathrm{B} \alpha$. The various stimuli that activate $\mathrm{NF}-\kappa \mathrm{B}$ cause phosphorylation of $\mathrm{I} \kappa \mathrm{B} \alpha$, which is followed by its ubiquitination and subsequent degradation. This results in the exposure of the nuclear localization signals on $\mathrm{NF}-\kappa \mathrm{B}$ subunits and the subsequent translocation of the molecule to the nucleus. Therefore, cell apoptosis due to knockdown of DDX46 could be explained, at least in part, by inhibition of the

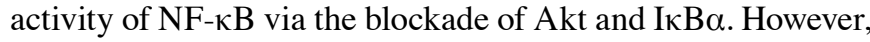
the direct link between DDX46 and the $\mathrm{NF}-\kappa \mathrm{B}$ signaling need to be investigated further.

In conclusion, our observations demonstrated that DDX46 was significantly upregulated in ESCC tissues and cell lines, DDX46 knockdown led to decreased proliferation and increased apoptosis in ESCC cells, which was mediated via decreased endogenous I $\mathrm{B} \alpha$ phosphorylation as well as negative regulation of $\mathrm{NF}-\kappa \mathrm{B}$ signaling. We hope that this study will provide the basis for novel strategy in the development of effective ESCC diagnosis and treatment. Further study is needed to clarify the specific molecular mechanisms for the role of DDX46 in the development and regulation of ESCC. 


\section{Acknowledgements}

This study was supported by a grant (No. 1308RJZA123) from the Natural Science Foundation of Gansu Province, China.

\section{References}

1. Torre LA, Bray F, Siegel RL, Ferlay J, Lortet-Tieulent J and Jemal A: Global cancer statistics, 2012. CA Cancer J Clin 65: 87-108, 2015.

2. Chen W, Zheng R, Zeng H, Zhang S and He J: Annual report on status of cancer in China, 2011. Chin J Cancer Res 27: 2-12, 2015.

3. Siewert JR and Ott K: Are squamous and adenocarcinomas of the esophagus the same disease? Semin Radiat Oncol 17: 38-44, 2007.

4. Gao YB, Chen ZL, Li JG, Hu XD, Shi XJ, Sun ZM, Zhang F, Zhao ZR, Li ZT, Liu ZY, et al: Genetic landscape of esophageal squamous cell carcinoma. Nat Genet 46: 1097-1102, 2014.

5. Moorcraft SY and Chau I: Investigational therapies targeting the ErbB family in oesophagogastric cancer. Expert Opin Investig Drugs 23: 1349-1363, 2014.

6. Jankowsky E: RNA helicases at work: Binding and rearranging. Trends Biochem Sci 36: 19-29, 2011.

7. Linder $P$ and Fuller-Pace F: Happy birthday: 25 years of DEAD-box proteins. Methods Mol Biol 1259: 17-33, 2015.

8. Fuller-Pace FV: DEAD box RNA helicase functions in cancer. RNA Biol 10: 121-132, 2013.

9. Heerma van Voss MR, Vesuna F, Trumpi K, Brilliant J, Berlinicke C, de Leng W, Kranenburg O, Offerhaus GJ, Bürger $\mathrm{H}$, van der Wall E, et al: Identification of the DEAD box RNA helicase DDX3 as a therapeutic target in colorectal cancer. Oncotarget 6: 28312-28326, 2015.

10. Bol GM, Vesuna F, Xie M, Zeng J, Aziz K, Gandhi N, Levine A, Irving A, Korz D, Tantravedi S, et al: Targeting DDX3 with a small molecule inhibitor for lung cancer therapy. EMBO Mol Med 7: 648-669, 2015.

11. $\mathrm{Xu} \mathrm{YZ} \mathrm{and} \mathrm{Query} \mathrm{CC:} \mathrm{Competition} \mathrm{between} \mathrm{the} \mathrm{ATPase} \mathrm{Prp5}$ and branch region-U2 snRNA pairing modulates the fidelity of spliceosome assembly. Mol Cell 28: 838-849, 2007.

12. Kosowski TR, Keys HR, Quan TK and Ruby SW: DExD/H-box Prp5 protein is in the spliceosome during most of the splicing cycle. RNA 15: 1345-1362, 2009

13. Hozumi S, Hirabayashi R, Yoshizawa A, Ogata M, Ishitani T, Tsutsumi M, Kuroiwa A, Itoh M and Kikuchi Y: DEAD-box protein Ddx46 is required for the development of the digestive organs and brain in zebrafish. PLoS One 7: e33675, 2012.

14. Hirabayashi R, Hozumi S, Higashijima S and Kikuchi Y: Ddx46 is required for multi-lineage differentiation of hematopoietic stem cells in zebrafish. Stem Cells Dev 22: 2532-2542, 2013.

15. Abdelhaleem M, Maltais L and Wain H: The human DDX and DHX gene families of putative RNA helicases. Genomics 81: 618-622, 2003.

16. Li M, Ma Y, Huang P, Du A, Yang X, Zhang S, Xing C, Liu F and Cao J: Lentiviral DDX46 knockdown inhibits growth and induces apoptosis in human colorectal cancer cells. Gene 560: 237-244, 2015

17. Linder P, Lasko PF, Ashburner M, Leroy P, Nielsen PJ, Nishi K, Schnier J and Slonimski PP: Birth of the D-E-A-D box. Nature 337: 121-122, 1989.

18. Germain DR, Graham K, Glubrecht DD, Hugh JC, Mackey JR and Godbout R: DEAD box 1: A novel and independent prognostic marker for early recurrence in breast cancer. Breast Cancer Res Treat 127: 53-63, 2011

19. Lu D and Yunis JJ: Cloning, expression and localization of an RNA helicase gene from a human lymphoid cell line with chromosomal breakpoint 11q23.3. Nucleic Acids Res 20: 1967-1972, 1992.

20. Akao Y, Marukawa O, Morikawa H, Nakao K, Kamei M, Hachiya $\mathrm{T}$ and Tsujimoto $\mathrm{Y}$ : The $\mathrm{rck} / \mathrm{p} 54$ candidate protooncogene product is a 54-kilodalton D-E-A-D box protein differentially expressed in human and mouse tissues. Cancer Res 55: 3444-3449, 1995.
21. Hashimoto K, Nakagawa Y, Morikawa H, Niki M, Egashira Y, Hirata I, Katsu K and Akao Y: Co-overexpression of DEAD box protein rck/p54 and c-myc protein in human colorectal adenomas and the relevance of their expression in cultured cell lines. Carcinogenesis 22: 1965-1970, 2001

22. Nakagawa Y, Morikawa H, Hirata I, Shiozaki M, Matsumoto A, Maemura K, Nishikawa T, Niki M, Tanigawa N, Ikegami M, et al: Overexpression of $\mathrm{rck} / \mathrm{p} 54$, a DEAD box protein, in human colorectal tumours. Br J Cancer 80: 914-917, 1999.

23. Miyaji K, Nakagawa Y, Matsumoto K, Yoshida H, Morikawa $H$, Hongou Y, Arisaka Y, Kojima H, Inoue T, Hirata I, et al: Overexpression of a DEAD box/RNA helicase protein, rck/ $\mathrm{p} 54$, in human hepatocytes from patients with hepatitis $\mathrm{C}$ virusrelated chronic hepatitis and its implication in hepatocellular carcinogenesis. J Viral Hepat 10: 241-248, 2003.

24. Causevic M, Hislop RG, Kernohan NM, Carey FA, Kay RA, Steele RJ and Fuller-Pace FV: Overexpression and polyubiquitylation of the DEAD-box RNA helicase p68 in colorectal tumours. Oncogene 20: 7734-7743, 2001.

25. Yang L, Lin C and Liu ZR: Phosphorylations of DEAD box p68 RNA helicase are associated with cancer development and cell proliferation. Mol Cancer Res 3: 355-363, 2005.

26. Shin S, Rossow KL, Grande JP and Janknecht R: Involvement of RNA helicases p68 and p72 in colon cancer. Cancer Res 67: 7572-7578, 2007.

27. Clark EL, Coulson A, Dalgliesh C, Rajan P, Nicol SM, Fleming S, Heer R, Gaughan L, Leung HY, Elliott DJ, et al: The RNA helicase p68 is a novel androgen receptor coactivator involved in splicing and is overexpressed in prostate cancer. Cancer Res 68: 7938-7946, 2008

28. Wortham NC, Ahamed E, Nicol SM, Thomas RS, Periyasamy M, Jiang J, Ochocka AM, Shousha S, Huson L, Bray SE, et al: The DEAD-box protein p72 regulates ERalpha-/oestrogen-dependent transcription and cell growth, and is associated with improved survival in ERalpha-positive breast cancer. Oncogene 28: 4053-4064, 2009.

29. Mazurek A, Luo W, Krasnitz A, Hicks J, Powers RS and Stillman B: DDX5 regulates DNA replication and is required for cell proliferation in a subset of breast cancer cells. Cancer Discov 2: 812-825, 2012.

30. Wang SJ, Zhang C, You Y and Shi CM: Overexpression of RNA helicase $\mathrm{p} 68$ protein in cutaneous squamous cell carcinoma. Clin Exp Dermatol 37: 882-888, 2012.

31. Wang R, Jiao Z, Li R, Yue H and Chen L: p68 RNA helicase promotes glioma cell proliferation in vitro and in vivo via direct regulation of NF- $\kappa \mathrm{B}$ transcription factor $\mathrm{p} 50$. Neuro-oncol 14: 1116-1124, 2012.

32. Sugiura T, Nagano Y and Noguchi Y: DDX39, upregulated in lung squamous cell cancer, displays RNA helicase activities and promotes cancer cell growth. Cancer Biol Ther 6: 957-964, 2007.

33. Kikuta K, Kubota D, Saito T, Orita H, Yoshida A, Tsuda H, Suehara Y, Katai H, Shimada Y, Toyama Y, et al: Clinical proteomics identified ATP-dependent RNA helicase DDX39 as a novel biomarker to predict poor prognosis of patients with gastrointestinal stromal tumor. J Proteomics 75: 1089-1098, 2012.

34. Lin F, Wang R, Shen JJ, Wang X, Gao P, Dong K and Zhang HZ: Knockdown of RCK/p54 expression by RNAi inhibits proliferation of human colorectal cancer cells in vitro and in vivo. Cancer Biol Ther 7: 1669-1676, 2008

35. Nicol SM, Bray SE, Black HD, Lorimore SA, Wright EG, Lane DP, Meek DW, Coates PJ and Fuller-Pace FV: The RNA helicase p68 (DDX5) is selectively required for the induction of p53-dependent p21 expression and cell-cycle arrest after DNA damage. Oncogene 32: 3461-3469, 2013

36. Testa JR and Bellacosa A: AKT plays a central role in tumorigenesis. Proc Natl Acad Sci USA 98: 10983-10985, 2001.

37. Mandal M, Kim S, Younes MN, Jasser SA, El-Naggar AK, Mills GB and Myers JN: The Akt inhibitor KP372-1 suppresses Akt activity and cell proliferation and induces apoptosis in thyroid cancer cells. Br J Cancer 92: 1899-1905, 2005.

38. Fresno Vara JA, Casado E, de Castro J, Cejas P, Belda-Iniesta C and González-Barón M: PI3K/Akt signalling pathway and cancer. Cancer Treat Rev 30: 193-204, 2004. 